\title{
Basic Study on Personal Space while Using Mobile Devices in Public
}

\author{
Shigeyoshi Iizuka ${ }^{1}$ and Kentaro $\mathrm{Go}^{2}$ \\ ${ }^{1}$ Kanagawa University, Japan \\ shigeiizuka@gmail.com \\ ${ }^{2}$ Interdisciplinary Graduate School of Medicine and Engineering, \\ University of Yamanashi, Japan \\ go@yamanashi.ac.jp
}

\begin{abstract}
In recent years, smart mobile devices have come to be widely used in our life spaces. This development has the potential to create major changes in the ways people communicate in public spaces. This paper presents two studies on a user's personal space while using a device in public: the first investigated mobile device use between three people; the second examined communicating private information on large, interactive displays in public spaces. Then, we introduced the basic concept for our future research on personal space while using mobile devices in public.
\end{abstract}

Keywords: personal space, public space, mobile devices, security.

\section{Introduction}

Today, information can be communicated regardless of time or place given the ubiquity of computers and the development of the Internet. Mobile devices in particular have come to be widely used in our life spaces in recent years, significantly influencing our activities. Therefore, it is thought that mobile devices will produce major changes to the ways we communicate in public spaces.

This paper presents two studies on personal space while using devices in public: the first investigated mobile device use between three people; the second examined the use of large public displays. Then, we introduce our basic concept on personal space research in the interactive display experience in public spaces; this will be the developed theme for our future research.

\section{Observation of Mobile Device Use between Three People}

To understand communication between two or more people while sharing a smart mobile device, we conducted an experiment consisting of three kinds of devicesharing methods (Iizuka et al., 2010). The purpose of this approach was to investigate 
the spatial relationships among the participants in each method of conversation using a smart mobile device.

Specifically, the following three methods were devised to share visual content on the display of a smart mobile device:

1. A device is passed mutually between the participants (mutually passed condition).

2. All participants look at content on a device that one person holds (device fixed to one person condition).

3. No one has a device during the experiment, but they talk about content seen beforehand (no device condition).

The participants were arranged into three-person groups; where possible, the group members were acquaintances. The experiment was conducted separately for each group.

Under each of the three above-mentioned conditions, each group used a small PC (VAIO type UX) (Figure 1) with a 4.5-inch display as a device shared between three people. Each group was directed to talk for two minutes about the picture displayed on the device (one picture was used for each condition; each picture was chosen at random from three images, as shown in Figure 2). The instructions given to the participants for each condition are shown in Table 1.

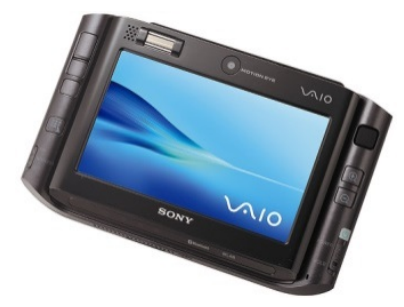

Fig. 1. Device for experiment
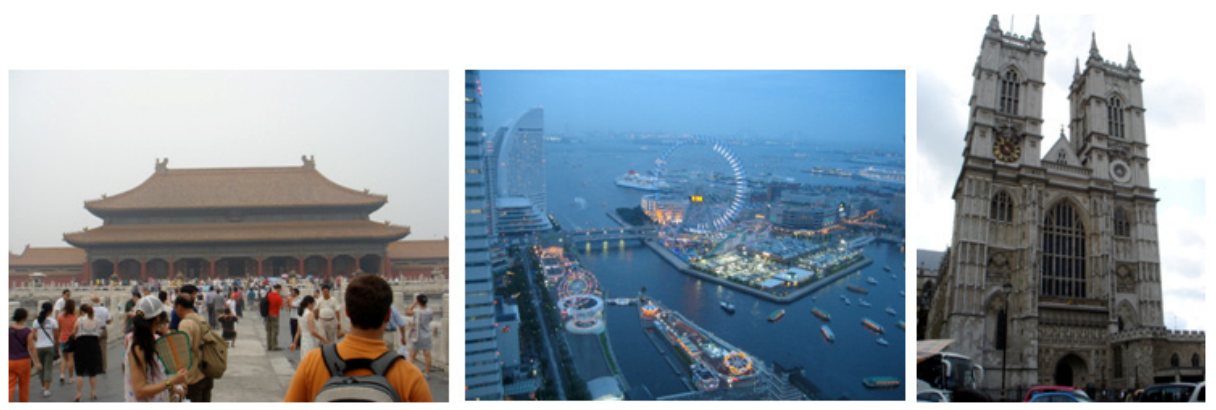

Fig. 2. Presentation images 
Table 1. Instructions for the experiment

\begin{tabular}{l|l}
\hline \multicolumn{1}{c|}{ Condition } & \multicolumn{1}{c}{ Instruction } \\
\hline Mutually passed & $\begin{array}{l}\text { Please talk for about two minutes while looking at } \\
\text { the picture on the device. Please pass the device } \\
\text { around and show the image to each other. }\end{array}$ \\
\hline Device fixed to one person & $\begin{array}{l}\text { Please talk for about two minutes while looking at } \\
\text { the picture on the device. Please have one person } \\
\text { hold the device while the others look on. }\end{array}$ \\
\hline No device & $\begin{array}{l}\text { Please view the picture first and then talk about it for } \\
\text { approximately two minutes. }\end{array}$ \\
\hline
\end{tabular}

The experimental environment was designed in a conference room at a university. A grid (60 cm spacing, three meters per side) was created on the floor using tape (Figure 3).

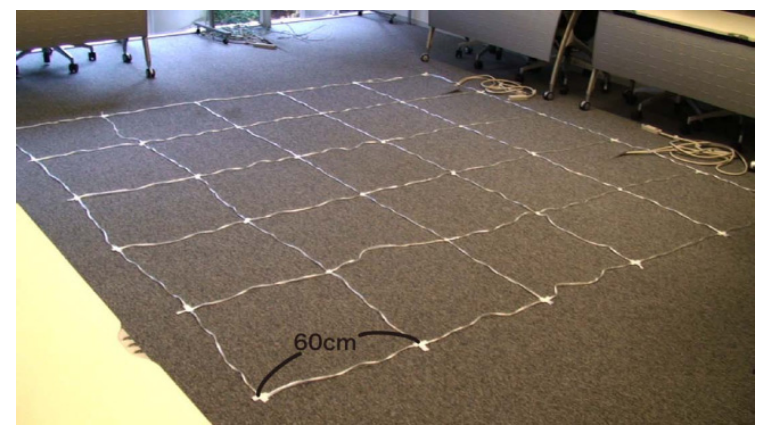

Fig. 3. Experimental grid

Before starting each trial, the three participants were gathered into one place to reset their positions. During each experiment, all participants were allowed to move freely within the experimental grid. All experiments were recorded with a video camera. After the trials, we visually plotted the positions of each participant during each two-minute experiment using the grid in the video image. A sample result is shown in Figure 4.

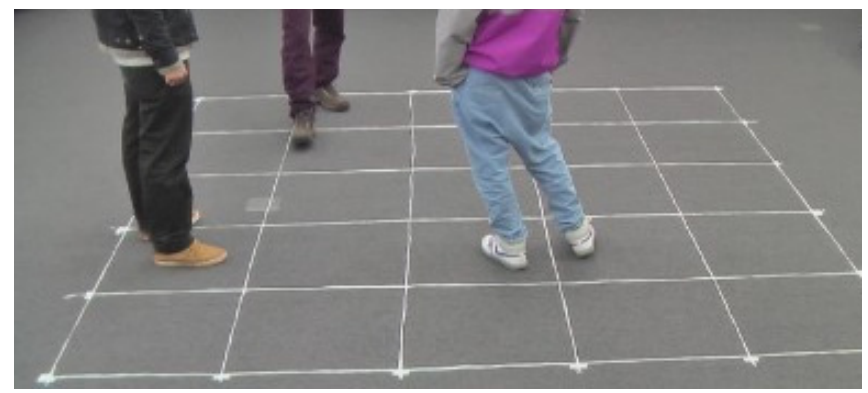

Fig. 4. The situation under experiment 
Here, we paid attention to the form of the triangle created by the positions of the three participants (Figure 5). The arrows in the figures indicate both the position and direction of each participant's step.
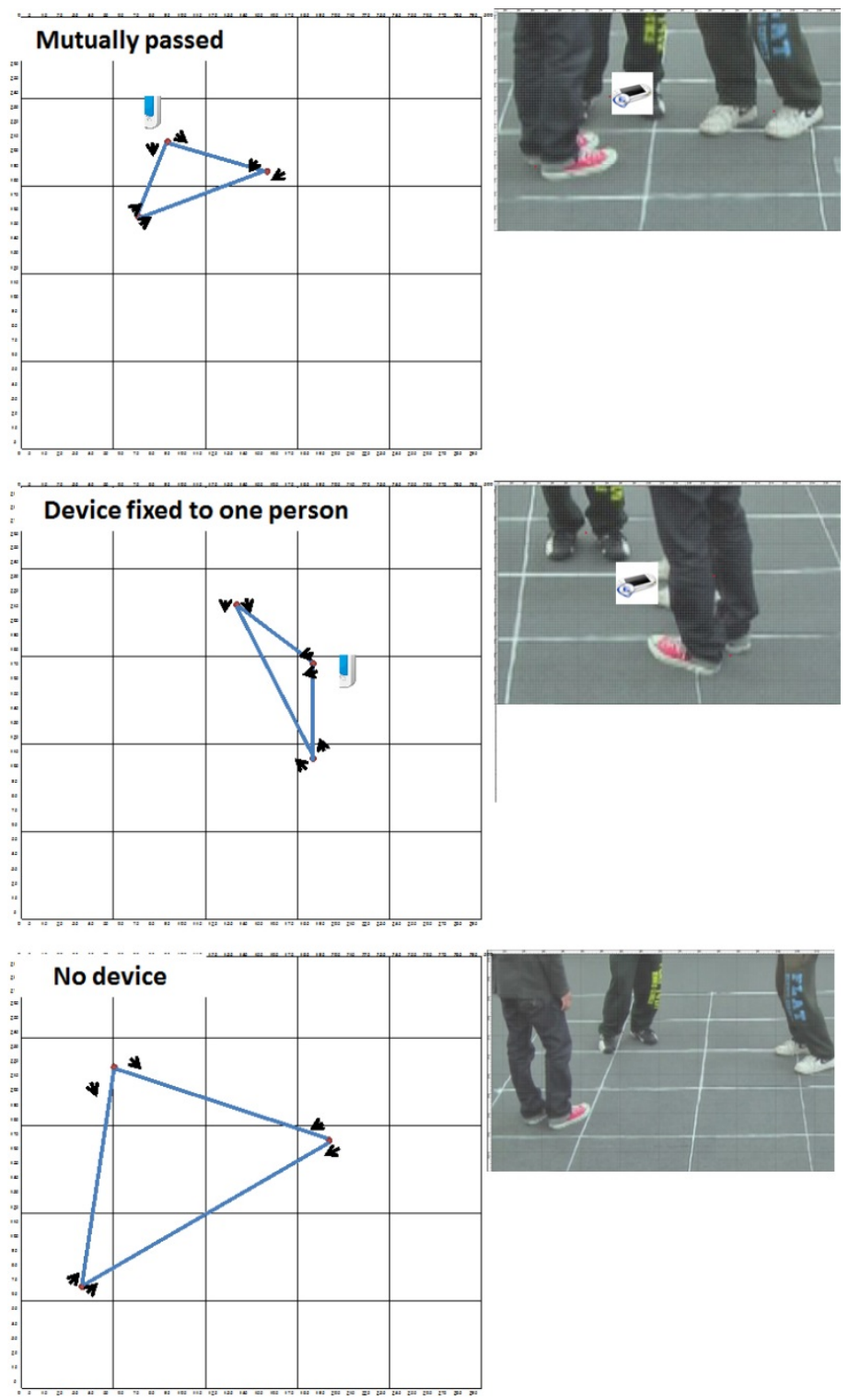

Fig. 5. Relationships between participant positions

In the "no device" and "mutually passed" conditions, the participants" positions formed equilateral triangles. The "device fixed to one person" condition, however, produced a number of obtuse isosceles triangles. 


\section{Examination of Large Public Display Use}

In public spaces such stations and shopping malls, the practical use of large displays is increasing. Since large-screen displays can provide information dynamically, they are very effective for simultaneously providing information to several people. As such, they are used for time guidance in train stations, navigational guidance around stations, or goods guidance at shopping malls.

Some digital signage terminals not only dispatch information in one direction but also have interactive interfaces. With such an interface, the user may freely access via touch various kinds of information (e.g., a map, store, or advertisement). Many user interfaces with touch panels have been utilized in public spaces, facilitating an interactive exchange of information (Figure 6).

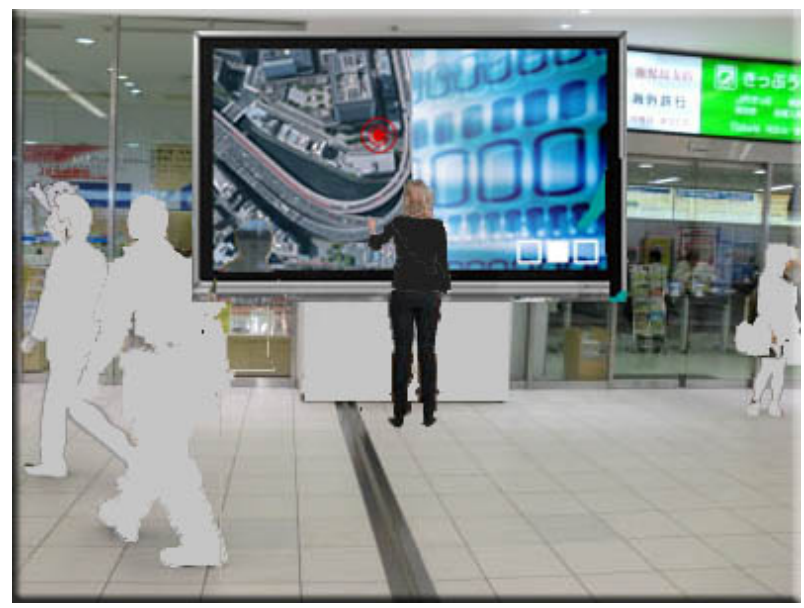

Fig. 6. Large public display use

Therefore, we studied information security and privacy for large touch screens in public spaces. We verified the validity of showing the information on peripheral danger to the user (Iizuka et al., 2014). Specifically, we conducted experiments using the following two information presentation methods:

- The user can check the situation behind him or her via a video of his or her back shown on the public display.

- The user is gradually notified of the grade of the danger via color information on the public display.

First, we developed a prototype system for the experiment. To gradually notify the user of danger, it was necessary to demarcate the space in front of the display according to distance. While a similar method has already been proposed by Vogel and others [1], in our model, the interaction of the user using the domain in front of the display does not change, and the divided domain is used as an index to notify the user 
of danger (Figure 7). Based on the distance (domain) from the display, the system gradually notifies the user of danger using color information (Figure 8):

- An arrow displays on the passing person in the image.

- A color frame displays on the outer frame of the user's workplace window.

- Both an arrow and a color frame are displayed.

- With no system

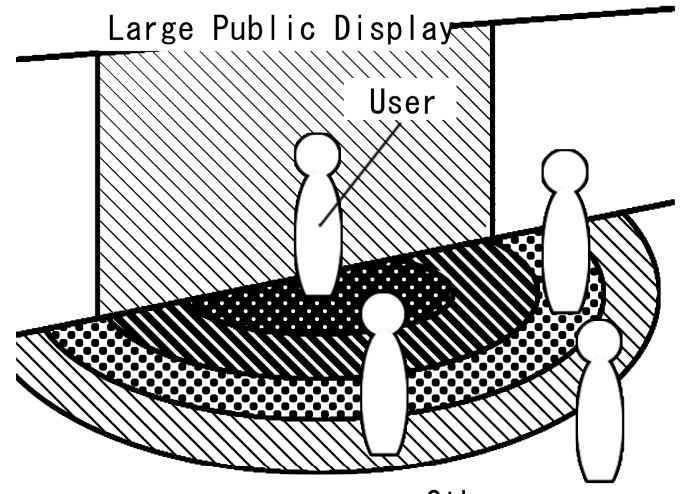

Others

Fig. 7. The domain in front of the display

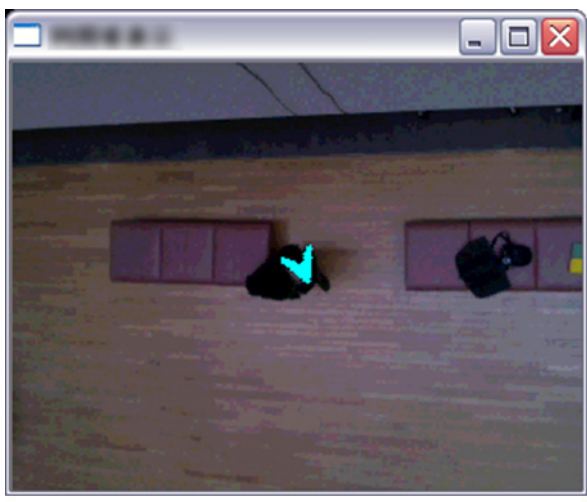

An arrow displays on the passing person in the image.

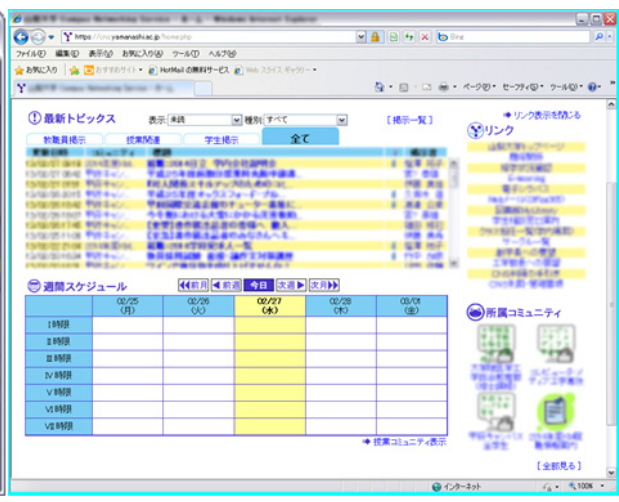

A color frame is displayed on the outer frame of the user's workplace window.

Fig. 8. Displaying information for users

The results found that notifying the user of "danger" did not necessarily reassure the user and could cause unease. It was suggested that displaying both an arrow indicating the position and direction of the passing person and a color frame based on the situation behind the user was more reassuring. 


\section{The Future of Personal Space within Public Spaces}

The increasing ubiquity of services is expected to make computing all the more prevalent in public spaces. A great deal of digital signage has been installed in public areas, and displays that present information interactively are also increasing. Furthermore, people in public possess personal devices such as smart phones. People in public spaces communicate various information, and use their devices properly according to the situation.

However, the transmission of information in public spaces is greatly influenced by spatial relationships involving others who share the same environment. Therefore, new designs are required in consideration of the following three elements: "place," "device (information)," and "person." Harmony between these three elements is necessary-that is, the design of the device-use environment should be adapted to the place, and there needs to be a balance between the information user and others in the surrounding public space.

It is believed the concept of "personal space" originated from the "spacing" (leaving space between individuals) of ethology. "Personal space" was studied by Sommer as one index of people's space behavior and was defined as "a domain of a certain size demarcated by an invisible boundary line" that surrounds an individual, into which others may not come (Sommer, 1959). Personal space affects individual actions and regulates relations between people. People are generally comfortable when their personal space is maintained and uncomfortable when others invade it (Shibuya, 1985). Furthermore, personal space is elastic and rarely fixed. Elasticity is also seen in relation to inanimate objects (IT equipment). Harada discusses people's displeasure over the use of a cellular phone by another person based on the concept of personal space (Harada, 1997). People who share physical space with a person talking on the phone feel the personal space of the person on the phone has spread. This creates a sense of incongruity, and people suddenly feel the talker's "space" cannot be entered. This causes displeasure. In other words, IT equipment and the transmission of information have the effect of expanding one person's personal space and contracting another's.

When information is communicated in a public space, it usually happens in the presence of strangers. In such situations, the environment inevitably has an effect on feelings of reassurance about one's personal space in relation to others.

As mentioned above, personal space might also change according to the situation at a given time, the nature of the service, or the contents of the transmitted information (Knowles, 1976). We plan, therefore, to prepare two or more experimental scenarios, set up environments based on their respective features, and develop the idea of personal space through user evaluation (Figure 9).

Furthermore, following the basic experiment that explored the new style of information sharing and personal communication using a mobile device, we plan to conduct user evaluations that use similar scenarios supposing two or more use scenes (Figure 10) and introduce knowledge. 


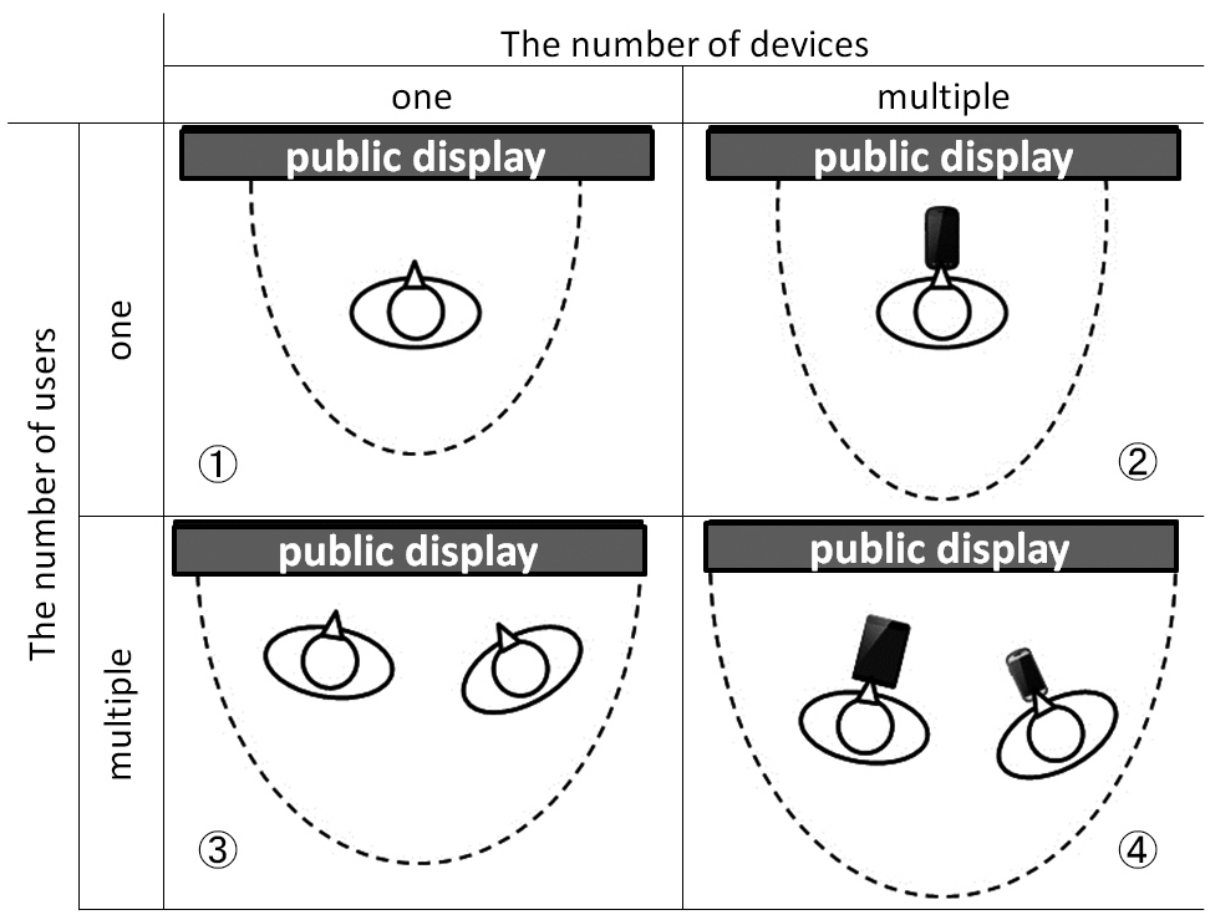

※Overhead view -the area inside the dotted line denotes personal space

Fig. 9. Use patterns of public display and mobile devices

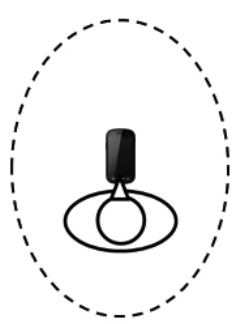

(1) One user uses one personal device.

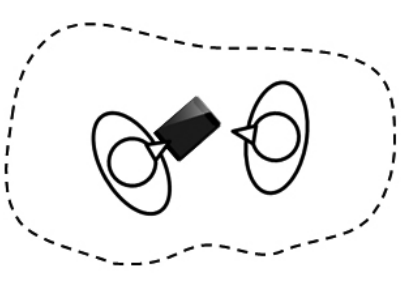

(2) Multiple users use one personal device.

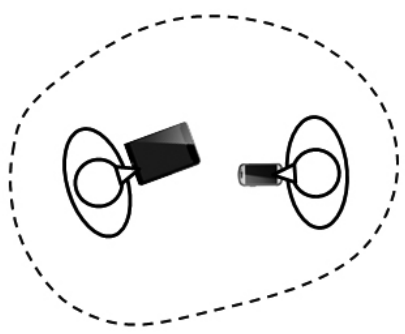

(3) Multiple users use multiple devices.

\section{※Overhead view - the area inside the dotted line denotes personal space}

Fig. 10. Patterns specialized in mobile device use

\section{Conclusion}

We focused first on smart mobile devices, which are widely used in our life spaces and may greatly change how we communicate in public spaces. We presented the results of an experiment that observed communication between three people under 
different conditions. Then, we discussed an experiment that involved displaying information on danger within the periphery of the user.

In future research, we aim to introduce a design that facilitates user comfort in communicating information in public spaces by applying the concept of personal space to communicating with interactive public displays or using mobile devices. Furthermore, we aim to develop a design indicator on the information environment in the public space to facilitate harmony with others in the surrounding space.

In the near future, with the expanded use of smart phones and tablets, communication through mobile devices in public spaces will increase significantly, which will have a still bigger influence on the information-transmission activities of people in public spaces. It is further expected that new styles of personal communication and information sharing will be generated. Research in this area should develop designs for transmitting information in ways that are suitable for this new style of communication in public spaces.

\section{References}

1. Harada, E.: Inanimate Object Research Seen from Human's Viewpoint. KyorituShuppan, Tokyo (1997) (in Japanese)

2. Iizuka, S., Kodama, A., Ogawa, K., Yasumura, M.: Aspect of Neighbor Communication at the time of Sharing a Smart Mobile Device. In: The 2nd Workshop on Human Centered Design, Human Centered Design Organization, pp. 3-4 (2010) (in Japanese)

3. Iizuka, S., Naito, W., Go, K., Kinoshita, Y.: A Study for Presentation Method of Information about Circumference Situation for Personal Use of Interactive Large Public Display. IPSJ Journal, IPS Japan (to be appeared, 2014) (in Japanese)

4. Knowles, E.S., Kreuser, B., Haas, H.M., Schuchart, G.E.: Group size and the extension of social space boundaries. Journal of Personality and Social Psychology 33, 647-654 (1976)

5. Shibuya, S.: Comfortable Distance between People. NHK Books, Tokyo (1990) (in Japanese)

6. Sommer, R.: Studies in Personal Space. Sociometry 22, 247-260 (1959) 